\title{
Tight asymptotic key rate for the BB84 protocol with local randomisation and device imprecisions
}

\author{
Erik Woodhead* \\ Laboratoire d'Information Quantique, CP 225, Université libre de Bruxelles, \\ av. F. D. Roosevelt 50, 1050 Bruxelles, Belgium
}

(Dated: 4 August 2014)

\begin{abstract}
Local randomisation is a preprocessing procedure in which one of the legitimate parties of a quantum key distribution (QKD) scheme adds noise to their version of the key and was found by Kraus et al. [Phys. Rev. Lett. 95, 080501 (2005)] to improve the security of certain QKD protocols. In this article, the improvement yielded by local randomisation is derived for an imperfect implementation of the BB84 QKD protocol, in which the source emits four given but arbitrary pure states and the detector performs arbitrarily-aligned measurements. Specifically, this is achieved by modifying an approach to analysing the security of imperfect variants of the BB84 protocol against collective attacks, introduced in [Phys. Rev. A 88, 012331 (2013)], to include the additional preprocessing step. The previously known improvement to the threshold channel noise, from $11 \%$ to $12.41 \%$, is recovered in the special case of an ideal BB84 implementation and becomes more pronounced in the case of a nonideal source. Finally, the bound derived for the asymptotic key rate, both with and without local randomisation, is shown to be tight with the particular source characterisation used. This is demonstrated by the explicit construction of a family of source states and optimal attacks for which the key-rate bound is attained with equality.
\end{abstract}

\section{INTRODUCTION}

Quantum key distribution (QKD) [1] was proposed three decades ago as a potentially feasible way to generate and distribute cryptographic keys in a secure manner, based on limitations inherent to quantum physics. The possibility of secure QKD intuitively follows from the monogamy of entanglement of quantum states or the impossibility of perfect state cloning [2,3], depending on the implementation.

The main theoretical problem in QKD consists in determining how many key bits can be extracted securely from a given protocol, i.e., determining a bound on the key rate, particularly under realistic conditions such as the presence of channel noise and imperfectly precise sources and detectors. This is an incompletely solved problem even for the original protocol proposed by Bennett and Brassard [1], now commonly called the BB84 protocol. For instance, in the case of an otherwise ideal implementation suffering from channel noise, and if the key is extracted using one-way postprocessing, the BB84 protocol has been shown to be secure asymptotically if the error rate is less than the Shor-Preskill error rate of $11 \%$ [4], but has only been proved insecure if the error rate exceeds around $14.64 \%$ [5]. Kraus et al. found that the lower threshold error rate could be increased to around $12.41 \%$ by local randomisation, in which one of the legitimate parties randomly flips a fraction of their raw key bits as a preprocessing step [6, 7]; Smith et al. subsequently increased this threshold to $12.92 \%$ using block codes [8]. If two-way postprocessing is used, the threshold error rate is known to lie between $20 \%$ and

\footnotetext{
*Erik.Woodhead@ulb.ac.be
}

$25 \%[9,10]$. More recently, local randomisation has been investigated for the BB84 protocol in the case of finite statistics [11].

Parallel to this, substantial effort has gone toward adapting security proofs for the BB84 protocol to account for device imprecisions, as described for instance in Refs. [12-18], where the goal is to derive a key-rate bound in a setting where either the source or measurement basis states or both are allowed to deviate from the $\sigma_{z}$ and $\sigma_{x}$ eigenstates ideally required by the BB84 protocol. Typically, the result is a generalisation of the Shor-Preskill key rate depending on additional parameters characterising the quantum devices, and the improvement obtainable with preprocessing is not investigated.

In this article, an approach to accounting for source imprecisions for the BB84 protocol, described in Ref. [18], is extended to include the local randomisation preprocessing procedure described in $[6,7]$. The approach is based on the security framework by Devetak and Winter [19] and can be used to derive bounds on the asymptotic key rate secure against an adversary restricted to collective attacks [20]. Conceptually, it separates into two steps: first, a lower bound on the Devetak-Winter key rate is derived in terms of an adversary's ability to distinguish their marginals of the $z$-basis states, as measured by the fidelity; second, the fidelity itself is lower bounded in terms of the observed error rate (such a bound can be viewed as quantifying the measurementdisturbance tradeoff or the limits on state cloning imposed by quantum physics). The incorporation of local randomisation presented here concerns only the first of these steps and is achieved by a straightforward generalisation of the intermediate bound on the key rate in terms of the fidelity derived in [18]. The approach can therefore automatically apply to any BB84-like protocol for which a fidelity bound of the type derived in [18] is 
known or can be derived.

Following this adaptation, particular attention is given to a nonideal BB84 implementation in which one party (Alice) has a source transmitting four characterised but arbitrary pure states and the second party (Bob)'s measurements are uncharacterised. The relative improvement obtained with the additional preprocessing is found to become more significant in the case of an imperfect source. Finally, the bound obtained on the Devetak-Winter rate, both with and without the additional preprocessing, is shown to be tight; this is demonstrated by the explicit construction of a family of sources and optimal collective attacks for which the Devetak-Winter rate is attained with equality.

As mentioned above, the security results derived here assume an adversary restricted to collective attacks. In Appendix A, we outline how the (prepare-and-measure) BB84 protocol considered here could be recast in an equivalent entanglement-based form, for which security against collective attacks is already known to imply security against general attacks under the assumption of a dimension bound on the Hilbert space.

\section{GENERAL SCENARIO AND METHOD}

The following generic setting is considered: one party (Alice) possesses a source capable of emitting one of a number of quantum states and transmitting them over an untrusted quantum channel to a second party (Bob), who performs measurements on them. Two of the states, which we will call the " $z$-basis" states (even if they are not orthogonal) and denote by $|\alpha\rangle$ and $\left|\alpha^{\prime}\right\rangle$, are intended for key generation. These should be selected (equiprobably between them) by Alice and transmitted to Bob the majority of the time. The remaining states are intended for the purpose of characterising the quantum channel and testing for the presence of an eavesdropper. One of Bob's possible measurements (a " $z$-basis" measurement) should be used a majority of the time and should ideally be calibrated in such a way that the measurement outcome is maximally correlated with Alice's choice of $z$ basis state. After all the quantum states have been transmitted, Alice and Bob publicly reveal in which cases they each used the $z$-basis states and measurement. A subset of the results are sacrificed in order to estimate the $z$ basis error rate, which we denote by $\delta_{z}$, after which they are discarded. The cases where at least one of Alice or Bob did not use the $z$ basis are used to estimate one or more parameters, which we collectively denote $\boldsymbol{\omega}$, depending on the specific details of the protocol. Alice then flips a fraction $\eta$ of her $z$-basis key bits, publicly revealing $\eta$ but not which bits she flips. Finally, Alice and Bob extract a key using one-way error correction and privacy amplification, as usual.

We assume that an adversary attacks the quantum channel unitarily, individually, identically, in which case they acquire partial traces $\rho_{\mathrm{E}}=\operatorname{Tr}_{\mathrm{B}}[|\alpha\rangle\langle\alpha|]$ or $\rho_{\mathrm{E}}^{\prime}=$
$\operatorname{Tr}_{\mathrm{B}}\left[\left|\alpha^{\prime}\right\rangle\left\langle\alpha^{\prime}\right|\right]$ of the $z$-basis states transmitted by Alice, and that they are allowed to delay their measurements indefinitely. In this case, a lower bound on the extractable secret key rate by one-way postprocessing is given by the Devetak-Winter rate [19], which we express as

$$
r=H(Z \mid \mathrm{E})-H\left(Z \mid Z^{\prime}\right) .
$$

In (1), $H\left(Z \mid Z^{\prime}\right)$ denotes the classical (Shannon) entropy of Alice's version of the key conditioned on Bob's $z$-basis measurement outcome and quantifies the key lost by error correction. In the typical case of symmetric errors, $H(Z \mid$ $\left.Z^{\prime}\right)=h\left(\tilde{\delta}_{z}\right)$, where $\tilde{\delta}_{z}=(1-\eta) \delta_{z}+\eta\left(1-\delta_{z}\right)$ is the error rate between Alice and Bob's $z$-basis results (after Alice has flipped a fraction $\eta$ of her bits), $h(p)=-p \log (p)-$ $(1-p) \log (1-p)$ is the binary entropy, and throughout this article, log is the base- 2 logarithm.

$H(Z \mid \mathrm{E})$ denotes the von Neumann entropy of Alice's key bits conditioned on the adversary's quantum side information, formally evaluated on the classical-quantum state

$$
\begin{aligned}
\tau_{Z \mathrm{E}}= & \frac{1}{2}\left((1-\eta)|0\rangle\left\langle\left. 0\right|_{Z}+\eta \mid 1\right\rangle\left\langle\left. 1\right|_{Z}\right) \otimes \rho_{\mathrm{E}}\right. \\
& +\frac{1}{2}\left(\eta|0\rangle\left\langle\left. 0\right|_{Z}+(1-\eta) \mid 1\right\rangle\left\langle\left. 1\right|_{Z}\right) \otimes \rho_{\mathrm{E}}^{\prime} .\right.
\end{aligned}
$$

In this framework, the main objective is to obtain a lower bound on $H(Z \mid \mathrm{E})$ in terms of the parameters $\boldsymbol{\omega}$ observed by Alice and Bob. Following the approach in [18], this can be separated into two steps. We first derive a lower bound on $H(Z \mid \mathrm{E})$ in terms of the fidelity $F\left(\rho_{\mathrm{E}}, \rho_{\mathrm{E}}^{\prime}\right)=\left\|\sqrt{\rho_{\mathrm{E}}} \sqrt{\rho_{\mathrm{E}}^{\prime}}\right\|_{1}$ of the adversary's marginal $z$-basis states. This can then be complemented by a suitable bound $F\left(\rho_{\mathrm{E}}, \rho_{\mathrm{E}}^{\prime}\right) \geq F(\boldsymbol{\omega})$ on the fidelity itself. Such a bound will generally depend on the details of the specific protocol being considered and assumptions about the source states and/or Bob's measurements. For example, for an ideal BB84 implementation with no local randomisation, the Shor-Preskill key rate is recovered by combining $H(Z \mid \mathrm{E}) \geq 1-h\left(\frac{1}{2}+\frac{1}{2} F\left(\rho_{\mathrm{E}}, \rho_{\mathrm{E}}^{\prime}\right)\right)$ with $F\left(\rho_{\mathrm{E}}, \rho_{\mathrm{E}}^{\prime}\right) \geq\left|1-2 \delta_{x}\right|$, where $\delta_{x}$ is the $x$-basis error rate [18].

In order to derive a lower bound on $H(Z \mid \mathrm{E})$, we first reexpress the classical-quantum state (2) as

$$
\tau_{Z \mathrm{E}}=\frac{1}{2}|0\rangle\left\langle\left. 0\right|_{Z} \otimes \tilde{\rho}_{\mathrm{E}}+\frac{1}{2} \mid 1\right\rangle\left\langle\left. 1\right|_{Z} \otimes \tilde{\rho}_{\mathrm{E}}^{\prime},\right.
$$

where $\tilde{\rho}_{\mathrm{E}}=\operatorname{Tr}_{\mathrm{B}}[\tilde{\rho}]$ and $\tilde{\rho}_{\mathrm{E}}^{\prime}=\operatorname{Tr}_{\mathrm{B}}\left[\tilde{\rho}^{\prime}\right]$, and we have set

$$
\begin{gathered}
\tilde{\rho}=(1-\eta) \rho+\eta \rho^{\prime}, \\
\tilde{\rho}^{\prime}=\eta \rho+(1-\eta) \rho^{\prime},
\end{gathered}
$$

where $\rho=|\alpha\rangle\langle\alpha|$ and $\rho^{\prime}=\left|\alpha^{\prime}\right\rangle\left\langle\alpha^{\prime}\right|$ are the two $z$-basis states. The conditional von Neumann entropy, evaluated directly on the classical-quantum state (3), simplifies to

$$
H(Z \mid \mathrm{E})=1+\frac{1}{2}\left(S\left(\tilde{\rho}_{\mathrm{E}}\right)+S\left(\tilde{\rho}_{\mathrm{E}}^{\prime}\right)\right)-S\left(\frac{1}{2}\left(\rho_{\mathrm{E}}+\rho_{\mathrm{E}}^{\prime}\right)\right),
$$

with $S(\rho)=-\operatorname{Tr}[\rho \log (\rho)]$. Following the approach in [18], we use that $H(Z \mid \mathrm{E}) \geq H\left(Z \mid \mathrm{EE}^{\prime}\right)$ for any extension $\tau_{Z \mathrm{EE}^{\prime}}$ of the state (3) to a larger Hilbert space 
in order to replace $\rho_{\mathrm{E}}$ and $\rho_{\mathrm{E}}^{\prime}$ with purifications $|\psi\rangle$ and $\left|\psi^{\prime}\right\rangle$ chosen such that $F\left(\rho_{\mathrm{E}}, \rho_{\mathrm{E}}^{\prime}\right)=\left\langle\psi \mid \psi^{\prime}\right\rangle$. With this substitution,

$$
\begin{aligned}
H(Z \mid \mathrm{E}) \geq & 1+\frac{1}{2} S\left((1-\eta)|\psi\rangle\left\langle\psi|+\eta| \psi^{\prime}\right\rangle\left\langle\psi^{\prime}\right|\right) \\
& +\frac{1}{2} S\left(\eta|\psi\rangle\left\langle\psi|+(1-\eta)| \psi^{\prime}\right\rangle\left\langle\psi^{\prime}\right|\right) \\
& -S\left(\frac{1}{2}\left(|\psi\rangle\left\langle\psi|+| \psi^{\prime}\right\rangle\left\langle\psi^{\prime}\right|\right)\right) .
\end{aligned}
$$

The eigenvalues of the operator $(1-\eta)|\psi\rangle\left\langle\psi|+\eta| \psi^{\prime}\right\rangle\left\langle\psi^{\prime}\right|$ are easily found to be $\frac{1}{2} \pm \frac{1}{2} \sqrt{1-4 \eta(1-\eta)\left(1-\left|\left\langle\psi \mid \psi^{\prime}\right\rangle\right|^{2}\right)}$. Consequently,

$$
\begin{aligned}
& H(Z \mid \mathrm{E}) \geq 1-h\left(\frac{1}{2}+\frac{1}{2} F\left(\rho_{\mathrm{E}}, \rho_{\mathrm{E}}^{\prime}\right)\right) \\
& \quad+h\left(\frac{1}{2}+\frac{1}{2} \sqrt{1-4 \eta(1-\eta)\left(1-F\left(\rho_{\mathrm{E}}, \rho_{\mathrm{E}}^{\prime}\right)^{2}\right)}\right) .
\end{aligned}
$$

In Appendix B, the right-hand side of (8) is shown to be an increasing function of the fidelity. Given a lower bound $F\left(\rho_{\mathrm{E}}, \rho_{\mathrm{E}}^{\prime}\right) \geq F(\boldsymbol{\omega})$ on the fidelity, then, we obtain the analytic lower bound

$$
\begin{aligned}
r \geq & +h\left(\frac{1}{2}+\frac{1}{2} \sqrt{1-4 \eta(1-\eta)\left(1-F(\boldsymbol{\omega})^{2}\right)}\right) \\
& -h\left(\frac{1}{2}+\frac{1}{2} F(\boldsymbol{\omega})\right)-h\left((1-\eta) \delta_{z}+\eta\left(1-\delta_{z}\right)\right)
\end{aligned}
$$

for the key rate.

The best result for the key rate is obtained by maximising the right-hand side of (9) over $\eta$, which, if necessary, is readily done numerically. Typically, as the channel noise approaches the maximal threshold, the optimal fraction $\eta$ approaches $1 / 2$. In this regime, the behaviour of the key-rate bound can be studied by substituting $\eta=(1-\varepsilon) / 2$ and expanding the resulting expression in powers of $\varepsilon$. The result, to the first non-trivial order in $\varepsilon$, is

$$
r \gtrsim\left(-\frac{1-F(\boldsymbol{\omega})^{2}}{4 F(\boldsymbol{\omega})} \log \left(\frac{1+F(\boldsymbol{\omega})}{1-F(\boldsymbol{\omega})}\right)+\frac{\left(1-2 \delta_{z}\right)^{2}}{2 \ln (2)}\right) \varepsilon^{2},
$$

where $\ln$ is the natural logarithm. Consequently, threshold error rates can be obtained by identifying corresponding roots of the expression

$$
\left(1-F(\boldsymbol{\omega})^{2}\right) \ln \left(\frac{1+F(\boldsymbol{\omega})}{1-F(\boldsymbol{\omega})}\right)-2 F(\boldsymbol{\omega})\left(1-2 \delta_{z}\right)^{2}
$$

\section{RESULT FOR NONIDEAL BB84}

The technique described in the preceding section can, in principle, be applied to any BB84-like protocol for which a bound of the form $F\left(\rho_{\mathrm{E}}, \rho_{\mathrm{E}}^{\prime}\right) \geq F(\boldsymbol{\omega})$ for the fidelity is known or can be derived. In this section, we illustrate its application to a nonideal prepare-and-measure BB84 implementation, in which Alice's source emits four arbitrary but characterised pure states and Bob performs uncharacterised measurements, for which a suitable fidelity bound is already given in [18]. In this setting, Alice's source emits two states, which we call the " $x$-basis" states and denote $|\beta\rangle$ and $\left|\beta^{\prime}\right\rangle$, and Bob performs an " $x$-basis" measurement, in addition to the $z$-basis states $|\alpha\rangle$ and $\left|\alpha^{\prime}\right\rangle$ and $z$-basis measurement previously described. (We adopt this nomenclature even if the source states and measurements do not satisfy the ideal BB84 relations.) We assume Alice's source is characterised by an angular parameter $\theta$, defined in terms of the source states by

$$
\sqrt{1+|\sin (\theta)|}=\frac{1}{2}\left|\langle\alpha \mid \beta\rangle+\left\langle\alpha^{\prime} \mid \beta\right\rangle+\left\langle\alpha \mid \beta^{\prime}\right\rangle-\left\langle\alpha^{\prime} \mid \beta^{\prime}\right\rangle\right|,
$$

wherever the right-hand side of (12) is greater than 1 . Given this characterisation, the fidelity between Eve's marginals of the $z$ states is lower bounded by

$$
\begin{aligned}
F\left(\rho_{\mathrm{E}}, \rho_{\mathrm{E}}^{\prime}\right) \geq & |\sin (\theta)| D\left(\sigma_{\mathrm{B}}, \sigma_{\mathrm{B}}^{\prime}\right) \\
& -|\cos (\theta)| \sqrt{1-D\left(\sigma_{\mathrm{B}}, \sigma_{\mathrm{B}}^{\prime}\right)^{2}},
\end{aligned}
$$

where $\sigma_{\mathrm{B}}=\operatorname{Tr}_{\mathrm{E}}[|\beta\rangle\langle\beta|], \quad \sigma_{\mathrm{B}}^{\prime}=\operatorname{Tr}_{r E}\left[\left|\beta^{\prime}\right\rangle\left\langle\beta^{\prime}\right|\right]$, and $D\left(\sigma_{\mathrm{B}}, \sigma_{\mathrm{B}}^{\prime}\right)=\frac{1}{2}\left\|\sigma_{\mathrm{B}}-\sigma_{\mathrm{B}}^{\prime}\right\|_{1}$ is the trace distance between Bob's marginals of the $x$ states [18]. The trace distance itself is lower bounded by $D\left(\sigma_{\mathrm{B}}, \sigma_{\mathrm{B}}^{\prime}\right) \geq\left|1-2 \delta_{x}\right|$ in terms of the $x$-basis error rate, regardless of how Bob's measurement apparatus is oriented. Explicitly combining these with the generic key-rate expression (9), we obtain the bound

$$
\begin{aligned}
r \geq & 1+h\left(\frac{1}{2}+\frac{1}{2} \sqrt{1-4 \eta(1-\eta)\left(1-f_{\theta}\left(\left|1-2 \delta_{x}\right|\right)^{2}\right)}\right) \\
& -h\left(\frac{1}{2}+\frac{1}{2} f_{\theta}\left(\left|1-2 \delta_{x}\right|\right)\right)-h\left(\tilde{\delta}_{z}\right),
\end{aligned}
$$

with $\tilde{\delta}_{z}=(1-\eta) \delta_{z}+\eta\left(1-\delta_{z}\right)$ and $f_{\theta}$ defined by

$$
f_{\theta}(x)=\left\{\begin{array}{ll}
|\sin (\theta)| x-|\cos (\theta)| \sqrt{1-x^{2}} & : x \geq|\cos (\theta)| \\
0 & : x \leq|\cos (\theta)|
\end{array},\right.
$$

for the key rate with local randomisation applied. For $\eta=0$, we recover the key rate

$$
r \geq 1-h\left(\frac{1}{2}+\frac{1}{2} f_{\theta}\left(\left|1-2 \delta_{x}\right|\right)\right)-h\left(\delta_{z}\right)
$$

given in [18], which itself coincides with the rate derived in [15] in the setting under consideration here.

The rates (14) and (16) (with and without local randomisation, respectively) are illustrated for a few values of $\theta$ in Fig. 1, assuming symmetric errors (i.e., $\delta_{z}=\delta_{x}=$ $\delta$ ) for simplicity. The depicted rates with local randomisation were found by numerically maximising (14) over $\eta$. For $\theta=\pi / 2=90^{\circ}$, corresponding to an ideal BB84 source, we recover the Shor-Preskill rate [4] and the improvement with local randomisation depicted in Fig. 2 of Ref. [7].

The threshold error rates, i.e., the error rates for which the key rates (16) without preprocessing and (14) with optimal local randomisation become zero, again for $\delta_{z}=\delta_{x}=\delta$, are depicted in Fig. 2 as a function of $\theta$. The threshold curve with local randomisation was found by identifying the corresponding root of (11). For $\theta=\pi / 2=90^{\circ}$ we recover the threshold error rates of 


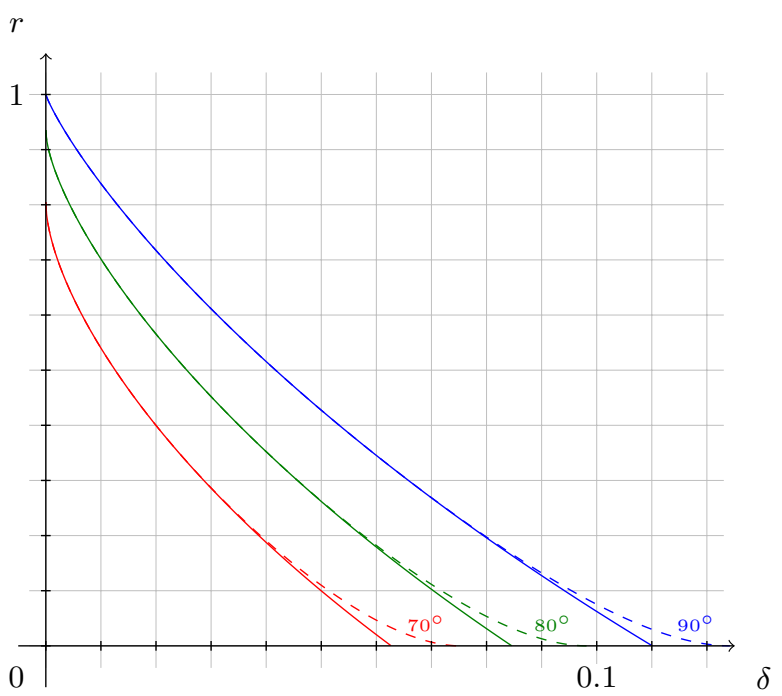

Figure 1. Key rates for $\theta=90^{\circ}, \theta=80^{\circ}$, and $\theta=70^{\circ}$ with (dashed curves) and without (solid curves) local randomisation, for $\delta=\delta_{z}=\delta_{x}$.

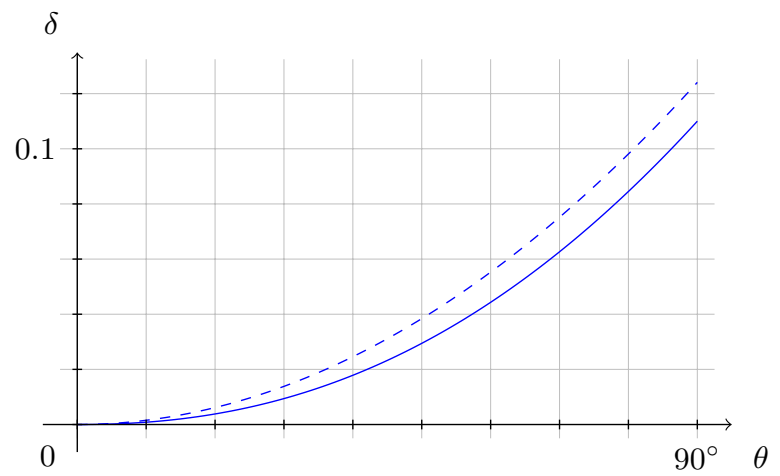

Figure 2. Threshold error rate with (dashed curve) and without (solid curve) local randomisation, for $0 \leq \theta \leq 90^{\circ}$.

$\delta \approx 12.4120 \%$ and $\delta \approx 11.0028 \%$ originally found in Refs. [6] and [4], respectively. For an ideal BB84 implementation, this corresponds to a relative increase of around $12.81 \%$ to the provably tolerable channel noise. This difference becomes more significant as $\theta$ decreases: for instance the relative improvement becomes around $20.00 \%$ ( $\delta \approx 7.5191 \%$ compared with $\delta \approx 6.2660 \%$ ) for $\theta=70^{\circ}$, around $33.84 \%(\delta \approx 3.1120 \%$ vs $\delta \approx 2.3251 \%)$ for $\theta=45^{\circ}$, and around $83.38 \%(\delta \approx 0.1538 \%$ vs $\delta \approx 0.08390 \%$ ) if $\theta$ is as low as $10^{\circ}$, indicating that the benefit of additional preprocessing becomes more pronounced for a realistic BB84 implementation expected to suffer from device imprecisions.

\section{OPTIMALITY OF KEY-RATE BOUND}

For the nonideal BB84 implementation considered in the preceding section, the key-rate bound (14) is tight in the sense that the Devetak-Winter rate (1) can be at- tained for all values of the independent variables $\theta, \delta_{z}$, $\delta_{x}$, and $\eta$. This is demonstrated here by the explicit construction of a family of source states and optimal unitary attacks. Equality between the right-hand sides of (1) and (14) requires the conditional von Neumann entropy bound (8) and the fidelity bound (13) to hold with equality simultaneously, which helps in the determination of an optimal attack. First, note that in the case of an equality, (13) rearranges to

$$
|\sin (\theta)|=F_{Z} D_{X}+\sqrt{1-F_{Z}^{2}} \sqrt{1-D_{X}^{2}},
$$

with $F_{Z}=F\left(\rho_{\mathrm{E}}, \rho_{\mathrm{E}}^{\prime}\right)$ and $D_{X}=D\left(\sigma_{\mathrm{B}}, \sigma_{\mathrm{B}}^{\prime}\right)$ and the condition $F_{Z} \leq D_{X}$. Equation (17) can equivalently be reexpressed as

$$
\begin{aligned}
\sqrt{1+|\sin (\theta)|}=\frac{1}{\sqrt{2}} & \left(\sqrt{1+F_{Z}} \sqrt{1+D_{X}}\right. \\
& \left.+\sqrt{1-F_{Z}} \sqrt{1-D_{X}}\right) .
\end{aligned}
$$

Consequently, our goal will be to construct source states such that the definition of the source characterisation (12) equals the right-hand side of (18).

Requiring $D\left(\rho_{\mathrm{B}}, \rho_{\mathrm{B}}^{\prime}\right)=D_{Z}$ suggests setting the $z$-basis states to the form

$$
\begin{aligned}
|\alpha\rangle & =\sqrt{\frac{1+D_{Z}}{2}}|0\rangle_{\mathrm{B}}\left|\psi_{0}\right\rangle_{\mathrm{E}}+\sqrt{\frac{1-D_{Z}}{2}}|1\rangle_{\mathrm{B}}\left|\psi_{1}^{\prime}\right\rangle_{\mathrm{E}}, \\
\left|\alpha^{\prime}\right\rangle & =\sqrt{\frac{1-D_{Z}}{2}}|0\rangle_{\mathrm{B}}\left|\psi_{1}\right\rangle_{\mathrm{E}}+\sqrt{\frac{1+D_{Z}}{2}}|1\rangle_{\mathrm{B}}\left|\psi_{0}^{\prime}\right\rangle_{\mathrm{E}},
\end{aligned}
$$

with $|0\rangle_{\mathrm{B}}$ and $|1\rangle_{\mathrm{B}}$ orthonormal. The trace distance $D\left(\rho_{\mathrm{B}}, \rho_{\mathrm{B}}^{\prime}\right)$ will equal $D_{Z}$ if $\left\langle\psi_{0} \mid \psi_{1}^{\prime}\right\rangle=\left\langle\psi_{1} \mid \psi_{0}^{\prime}\right\rangle=0$. In order for the fidelity $F\left(\rho_{\mathrm{E}}, \rho_{\mathrm{E}}^{\prime}\right)$ to equal $F_{Z}$, and in such a way that the von Neumann entropy bound (8) becomes an equality, we additionally require $\left\langle\psi_{0} \mid \psi_{1}\right\rangle=$ $\left\langle\psi_{0}^{\prime} \mid \psi_{1}^{\prime}\right\rangle=0$ and $\left\langle\psi_{0} \mid \psi_{0}^{\prime}\right\rangle=\left\langle\psi_{1} \mid \psi_{1}^{\prime}\right\rangle=F_{Z} \in \mathbb{R}^{+}$, such that $\left\{\left|\psi_{0}\right\rangle,\left|\psi_{0}^{\prime}\right\rangle\right\}$ and $\left\{\left|\psi_{1}\right\rangle,\left|\psi_{1}^{\prime}\right\rangle\right\}$ span two mutually orthogonal subspaces. Note that, with these definitions, $|\alpha\rangle$ and $\left|\alpha^{\prime}\right\rangle$ are normalised and orthogonal.

The right-hand side of the source characterisation (12) can be reexpressed as $\frac{1}{\sqrt{2}}\left|\left\langle\alpha_{+} \mid \beta\right\rangle+\left\langle\alpha_{-} \mid \beta^{\prime}\right\rangle\right|$ with $\left|\alpha_{ \pm}\right\rangle=$ $\frac{1}{\sqrt{2}}\left(|\alpha\rangle \pm\left|\alpha^{\prime}\right\rangle\right)$. Introducing, for convenience, the states

$$
\left|\alpha_{k}\right\rangle=|0\rangle_{\mathrm{B}}\left|\psi_{k}\right\rangle_{\mathrm{E}}, \quad\left|\alpha_{k}^{\prime}\right\rangle=|1\rangle_{\mathrm{B}}\left|\psi_{k}^{\prime}\right\rangle_{\mathrm{E}},
$$

and $\left|\alpha_{k}^{ \pm}\right\rangle=\frac{1}{\sqrt{2}}\left(\left|\alpha_{k}\right\rangle \pm\left|\alpha_{k}^{\prime}\right\rangle\right), k \in\{0,1\}$, we find

$$
\begin{aligned}
& \left|\alpha_{k}^{+}\right\rangle=\sqrt{\frac{1+F_{Z}}{2}}|+\rangle_{\mathrm{B}}\left|\psi_{k}^{+}\right\rangle_{\mathrm{E}}+\sqrt{\frac{1-F_{Z}}{2}}|-\rangle_{\mathrm{B}}\left|\psi_{k}^{-}\right\rangle_{\mathrm{E}} \\
& \left|\alpha_{k}^{-}\right\rangle=\sqrt{\frac{1-F_{Z}}{2}}|+\rangle_{\mathrm{B}}\left|\psi_{k}^{-}\right\rangle_{\mathrm{E}}+\sqrt{\frac{1+F_{Z}}{2}}|-\rangle_{\mathrm{B}}\left|\psi_{k}^{+}\right\rangle_{\mathrm{E}}
\end{aligned}
$$

where $| \pm\rangle_{\mathrm{B}}=\frac{1}{\sqrt{2}}\left(|0\rangle_{\mathrm{B}}+|1\rangle_{\mathrm{B}}\right)$ and the states

$$
\left|\psi_{k}^{ \pm}\right\rangle_{\mathrm{E}}=\frac{\left|\psi_{k}\right\rangle_{\mathrm{E}} \pm\left|\psi_{k}^{\prime}\right\rangle_{\mathrm{E}}}{\sqrt{2 \pm 2 F_{Z}}}
$$

are orthonormal. In terms of $\left|\alpha_{k}^{ \pm}\right\rangle$,

$$
\begin{aligned}
& \left|\alpha_{+}\right\rangle=\sqrt{\frac{1+D_{Z}}{2}}\left|\alpha_{0}^{+}\right\rangle+\sqrt{\frac{1-D_{Z}}{2}}\left|\alpha_{1}^{+}\right\rangle, \\
& \left|\alpha_{-}\right\rangle=\sqrt{\frac{1+D_{Z}}{2}}\left|\alpha_{0}^{-}\right\rangle-\sqrt{\frac{1-D_{Z}}{2}}\left|\alpha_{1}^{-}\right\rangle .
\end{aligned}
$$


It is then fairly straightforward to construct $x$-basis states for which the right-hand side of the source characterisation (12) will take the form of the right-hand side of (18). We set

$$
\begin{aligned}
& \left|\beta_{k}\right\rangle=\sqrt{\frac{1+D_{X}}{2}}|+\rangle_{\mathrm{B}}\left|\psi_{k}^{+}\right\rangle_{\mathrm{E}}+\sqrt{\frac{1-D_{X}}{2}}|-\rangle_{\mathrm{B}}\left|\psi_{k}^{-}\right\rangle_{\mathrm{E}}, \\
& \left|\beta_{k}^{\prime}\right\rangle=\sqrt{\frac{1-D_{X}}{2}}|+\rangle_{\mathrm{B}}\left|\psi_{k}^{-}\right\rangle_{\mathrm{E}}+\sqrt{\frac{1+D_{X}}{2}}|-\rangle_{\mathrm{B}}\left|\psi_{k}^{+}\right\rangle_{\mathrm{E}},
\end{aligned}
$$

and

$$
\begin{gathered}
|\beta\rangle=\sqrt{\frac{1+D_{Z}}{2}}\left|\beta_{0}\right\rangle+\sqrt{\frac{1-D_{Z}}{2}}\left|\beta_{1}\right\rangle, \\
\left|\beta^{\prime}\right\rangle=\sqrt{\frac{1+D_{Z}}{2}}\left|\beta_{0}^{\prime}\right\rangle-\sqrt{\frac{1-D_{Z}}{2}}\left|\beta_{1}^{\prime}\right\rangle .
\end{gathered}
$$

With these definitions we find

$$
\begin{aligned}
\left\langle\alpha_{+} \mid \beta\right\rangle=\left\langle\alpha_{-} \mid \beta^{\prime}\right\rangle=\frac{1}{2} & \left(\sqrt{1+F_{Z}} \sqrt{1+D_{X}}\right. \\
& \left.+\sqrt{1-F_{Z}} \sqrt{1-D_{X}}\right),
\end{aligned}
$$

independently of $D_{Z}$, from which we recover the righthand side of the rearrangement (18) of the fidelity bound (13).

Explicitly, from the expressions (19), (20), (29), and (30) for the $z$ - and $x$-basis states, Bob's marginals are given by

$$
\begin{aligned}
\rho_{\mathrm{B}} & =\frac{1+D_{Z}}{2}|0\rangle\left\langle\left. 0\right|_{\mathrm{B}}+\frac{1-D_{Z}}{2} \mid 1\right\rangle\left\langle\left. 1\right|_{\mathrm{B}},\right. \\
\rho_{\mathrm{B}}^{\prime} & =\frac{1-D_{Z}}{2}|0\rangle\left\langle\left. 0\right|_{\mathrm{B}}+\frac{1+D_{Z}}{2} \mid 1\right\rangle\left\langle\left. 1\right|_{\mathrm{B}},\right. \\
\sigma_{\mathrm{B}} & =\frac{1+D_{X}}{2}|+\rangle\left\langle+\left.\right|_{\mathrm{B}}+\frac{1-D_{X}}{2} \mid-\right\rangle\left\langle-\left.\right|_{\mathrm{B}},\right. \\
\sigma_{\mathrm{B}}^{\prime} & =\frac{1-D_{X}}{2}|+\rangle\left\langle+\left.\right|_{\mathrm{B}}+\frac{1+D_{X}}{2} \mid-\right\rangle\left\langle-\left.\right|_{\mathrm{B}} .\right.
\end{aligned}
$$

Consequently, Alice and Bob detect errors at the rates $\delta_{z}=\frac{1}{2}-\frac{1}{2} D_{Z}$ and $\delta_{x}=\frac{1}{2}-\frac{1}{2} D_{X}$ if Bob measures (optimally) in the $\sigma_{z}$ and $\sigma_{x}$ bases. Likewise, Eve's marginals of the $z$ states are given by

$$
\begin{gathered}
\rho_{\mathrm{E}}=\frac{1+D_{Z}}{2}\left|\psi_{0}\right\rangle\left\langle\left.\psi_{0}\right|_{\mathrm{E}}+\frac{1-D_{Z}}{2} \mid \psi_{1}^{\prime}\right\rangle\left\langle\left.\psi_{1}^{\prime}\right|_{\mathrm{E}},\right. \\
\rho_{\mathrm{E}}^{\prime}=\frac{1-D_{Z}}{2}\left|\psi_{1}\right\rangle\left\langle\left.\psi_{1}\right|_{\mathrm{E}}+\frac{1+D_{Z}}{2} \mid \psi_{0}^{\prime}\right\rangle\left\langle\left.\psi_{0}^{\prime}\right|_{\mathrm{E}},\right.
\end{gathered}
$$

for which one can readily verify that $F\left(\rho_{\mathrm{E}}, \rho_{\mathrm{E}}^{\prime}\right)=$ $\left\|\sqrt{\rho_{\mathrm{E}}} \sqrt{\rho_{\mathrm{E}}^{\prime}}\right\|_{1}=F_{Z}$ and, for any $p, q \geq 0$ and $p+q=1$,

$$
\begin{aligned}
S\left(p \rho_{\mathrm{E}}+q \rho_{\mathrm{E}}^{\prime}\right)= & h\left(\frac{1}{2}+\frac{1}{2} D_{Z}\right) \\
& +\frac{1+D_{Z}}{2} S\left(p\left|\psi_{0}\right\rangle\left\langle\left.\psi_{0}\right|_{\mathrm{E}}+q \mid \psi_{0}^{\prime}\right\rangle\left\langle\left.\psi_{0}^{\prime}\right|_{\mathrm{E}}\right)\right. \\
& +\frac{1-D_{Z}}{2} S\left(p\left|\psi_{1}^{\prime}\right\rangle\left\langle\left.\psi_{1}^{\prime}\right|_{\mathrm{E}}+q \mid \psi_{1}\right\rangle\left\langle\left.\psi_{1}\right|_{\mathrm{E}}\right)\right. \\
= & h\left(\frac{1}{2}+\frac{1}{2} D_{Z}\right) \\
& +h\left(\frac{1}{2}+\frac{1}{2} \sqrt{1-4 p q\left(1-F_{Z}^{2}\right)}\right) .
\end{aligned}
$$

Using (38) to directly evaluate the expression (6) for the conditional von Neumann entropy $H(Z \mid$ E), we find that its bound $(8)$ in terms of fidelity $F\left(\rho_{\mathrm{E}}, \rho_{\mathrm{E}}^{\prime}\right)$ is attained with equality for the entire family of sources and attacks just constructed.

Equations (19), (20), (29), and (30) give the optimal attack for a family of sources identified by the relations

$$
\left\langle\alpha \mid \alpha^{\prime}\right\rangle=\left\langle\beta \mid \beta^{\prime}\right\rangle=0
$$

and

$$
\langle\alpha \mid \beta\rangle=\left\langle\alpha^{\prime} \mid \beta\right\rangle=\left\langle\alpha \mid \beta^{\prime}\right\rangle=-\left\langle\alpha^{\prime} \mid \beta^{\prime}\right\rangle=\frac{\sqrt{1+|\sin (\theta)|}}{2},
$$

for which the bound (14) on the Devetak-Winter rate is attained with equality independently of the fraction $\eta$ of bits flipped by Alice in the local randomisation preprocessing step. The family of optimal attacks given here generalises the optimal individual attack derived for an ideal BB84 source in [5], which is recovered for $|\sin (\theta)|=1$ or, equivalently, by setting $F_{Z}=D_{X}$. Another extreme worth noting is the case $F_{Z}=0$ and $D_{Z}=D_{X}=1$, in which case $|\sin (\theta)|=0$ and

$$
\begin{array}{rlrl}
|\alpha\rangle=|0\rangle_{\mathrm{B}}|0\rangle_{\mathrm{E}}, & \left|\alpha^{\prime}\right\rangle & =|1\rangle_{\mathrm{B}}|1\rangle_{\mathrm{E}}, \\
|\beta\rangle & =|+\rangle_{\mathrm{B}}|+\rangle_{\mathrm{E}}, & \left|\beta^{\prime}\right\rangle & =|-\rangle_{\mathrm{B}}|+\rangle_{\mathrm{E}},
\end{array}
$$

i.e., the adversary acquires perfect copies of Bob's $z$-basis states without introducing any errors.

\section{CONCLUSION}

This article described how the local randomisation preprocessing technique proposed by Kraus et al. in $[6,7]$ can be incorporated into the security analysis introduced in [18] for the BB84 protocol. The improvement to the key rate and tolerable channel noise was explicitly quantified for an imperfect BB84 implementation in which Alice's source emits four arbitrary but characterised pure states and Bob's measurements are left largely uncharacterised. The improvement becomes more significant if the source is imperfect. The asymptotic key-rate bound (14) was shown to be tight given the source characterisation parameter $\theta$ defined in (12), and is attained for the family of source states and optimal collective attacks constructed in Sec. IV if Bob performs the ideal $\sigma_{z}$ and $\sigma_{x}$ measurements.

The setting described in Sec. II was left somewhat generic as the method is not necessarily limited to just the BB84 protocol itself. In particular it has already been found to apply to a semi-device-independent QKD protocol in which Alice's source and Bob's measurements are assumed two dimensional [21]. The device-independent protocol studied in [22] may also be a candidate; this is suggested by the fact that the conditional von Neumann entropy bound (8) is attained with equality for the optimal collective attack derived in [22].

\section{ACKNOWLEDGMENTS}

S. Pironio offered helpful comments on an early draft of this article. This work was supported by the EU projects Q-Essence and QAlgo, the CHIST-ERA DIQIP project, the Interuniversity Attraction Poles Photonics@be Programme (Belgian Science Policy), and the FRS-FNRS under project DIQIP. The author is supported by a Belgian Fonds pour la Formation à la Recherche 
dans l'Industrie et dans l'Agriculture (F.R.I.A.) doctoral grant.

\section{Appendix A: Equivalent entanglement-based protocol}

The main problem addressed in this article is the derivation of key rates for variants of the prepare-and-measure BB84 protocol secure against an adversary limited to collective attacks. In the case of entanglement-based QKD, security against collective attacks is known to imply security against general attacks in the asymptotic limit, at least under the assumption of a dimension bound, the assumption that Alice's and Bob's measurements are memoryless, and if a symmetrisation procedure is applied [23]. In this section, we briefly review how the BB84 protocol considered in Sec. III could be recast in the form of an equivalent entanglement-based protocol.

The starting point, already considered by the authors of [13-15], is that Alice could prepare the four source states by equivalently preparing and distributing an entangled "coin" state of the form

$$
\begin{aligned}
|\Psi\rangle_{\mathrm{ABE}}= & c_{00}|00\rangle_{\mathrm{A}}|\alpha\rangle_{\mathrm{BE}}+c_{01}|01\rangle_{\mathrm{A}}\left|\alpha^{\prime}\right\rangle_{\mathrm{BE}} \\
& +c_{10}|10\rangle_{\mathrm{A}}|\beta\rangle_{\mathrm{BE}}+c_{11}|11\rangle_{\mathrm{A}}\left|\beta^{\prime}\right\rangle_{\mathrm{BE}}
\end{aligned}
$$

and determining her bit and basis choice by measuring in the (orthonormal) $\left\{|00\rangle_{\mathrm{A}},|01\rangle_{\mathrm{A}},|10\rangle_{\mathrm{A}},|11\rangle_{\mathrm{A}}\right\}$ basis. In (A1), the coefficients $c_{i j}, i, j \in\{0,1\}$ determine the probability of Alice selecting a particular bit and basis and can always be taken to be real and nonnegative. In the setting considered in Sec. III, Alice uses the $z$ basis the majority of the time and chooses between the two states in each basis equiprobably. For the amplitudes $c_{i j}$, this translates to

$$
\begin{aligned}
c_{00}=c_{01} & =\sqrt{\frac{1-\varepsilon}{2}}, \\
c_{10}=c_{11} & =\sqrt{\frac{\varepsilon}{2}}
\end{aligned}
$$

for some given $\varepsilon$ close to zero. The states $|\alpha\rangle,\left|\alpha^{\prime}\right\rangle,|\beta\rangle,\left|\beta^{\prime}\right\rangle \in \mathcal{H}_{\mathrm{B}} \otimes \mathcal{H}_{\mathrm{E}}$ shared by Bob and Eve correspond to the $z$ - and $x$-basis states and the relations between them are fully specified, such that (A1) is determined up to an overall unitary on $\mathcal{H}_{\mathrm{B}} \otimes \mathcal{H}_{\mathrm{E}}$.

The virtual protocol described so far is not a secure entanglement-based protocol in the usual sense, as it requires Alice and Bob to trust that the shared entangled state is of the form given in (A1). To remove this, note that it is equivalent for Alice to know the marginal density operator $\rho_{\mathrm{A}}=\operatorname{Tr}_{\mathrm{BE}}\left[|\Psi\rangle\left\langle\left.\Psi\right|_{\mathrm{ABE}}\right]\right.$, as all purifications of $\rho_{\mathrm{A}}$ are related by unitaries on $\mathcal{H}_{\mathrm{B}} \otimes \mathcal{H}_{\mathrm{E}}$. Specifically, if $|\Psi\rangle_{\mathrm{ABE}}$ is any purification of $\rho_{\mathrm{A}}$, the $z$ and $x$ states and amplitutes appearing in (A1) can be recovered by

$$
\begin{aligned}
c_{00}|\alpha\rangle_{\mathrm{BE}} & =\left(\left\langle\left. 00\right|_{\mathrm{A}} \otimes \mathbb{1}_{\mathrm{BE}}\right)|\Psi\rangle_{\mathrm{ABE}},\right. \\
c_{01}\left|\alpha^{\prime}\right\rangle_{\mathrm{BE}} & =\left(\left\langle\left. 01\right|_{\mathrm{A}} \otimes \mathbb{1}_{\mathrm{BE}}\right)|\Psi\rangle_{\mathrm{ABE}},\right.
\end{aligned}
$$

$$
\begin{aligned}
c_{10}|\beta\rangle_{\mathrm{BE}} & =\left(\left\langle\left. 10\right|_{\mathrm{A}} \otimes \mathbb{1}_{\mathrm{BE}}\right)|\Psi\rangle_{\mathrm{ABE}},\right. \\
c_{11}\left|\beta^{\prime}\right\rangle_{\mathrm{BE}} & =\left(\left\langle\left. 11\right|_{\mathrm{A}} \otimes \mathbb{1}_{\mathrm{BE}}\right)|\Psi\rangle_{\mathrm{ABE}} .\right.
\end{aligned}
$$

Since $\rho_{\mathrm{A}}$ is reconstructable by tomography, Alice no longer needs to trust that the entangled state is of the form (A1), provided that she performs additional tomographic measurements to determine or verify that $\rho_{\mathrm{A}}$ is of a particular desired form as part of the virtual entanglement-based protocol outlined here.

\section{Appendix B: Convexity of conditional entropy bound}

The right-hand side of (8) has the form

$$
H(F)=1+\phi(R)-\phi(F),
$$

where, for convenience, we have set

$$
R=\sqrt{\lambda+\mu F^{2}},
$$

$\lambda=(1-2 \eta)^{2}$ and $\mu=4 \eta(1-\eta)$ (such that $0 \leq \lambda, \mu \leq 1$ and $\lambda+\mu=1)$, and the function $\phi$ is defined by

$$
\begin{aligned}
\phi(x)= & h\left(\frac{1}{2}+\frac{1}{2} x\right) \\
= & 1-\frac{1}{2}(1+x) \log (1+x) \\
& -\frac{1}{2}(1-x) \log (1-x)
\end{aligned}
$$

for $-1<x<1$ and $\phi(1)=\phi(-1)=0$.

If $\mu=1$ (and $\lambda=0$ ), (B1) reduces to $H(F)=1$. In the following we show that, for $\mu<1, H$ is a convex function by showing that its second derivative in $F$ is nonnegative. Since its global minimum is $H(0)=0$, it will follow that $H$ is an increasing function over the range $0 \leq F \leq 1$.

We first evaluate the first and second derivatives of $\phi$; respectively, they are

$$
\phi^{\prime}(x)=-\frac{1}{2} \log \left(\frac{1+x}{1-x}\right)
$$

and

$$
\phi^{\prime \prime}(x)=-\frac{1}{\ln (2)} \frac{1}{1-x^{2}} .
$$

For the first and second derivatives of $R$ (viewed as a function of $F$ ), we obtain $R^{\prime}=\mu F / R$ and $R^{\prime \prime}=\lambda \mu / R^{3}$. In terms of $\phi$ and its derivatives and $R$, the first and second derivatives of $H$ are

$$
H^{\prime}(F)=\phi^{\prime}(R) \frac{\mu F}{R}-\phi^{\prime}(F),
$$

and

$$
H^{\prime \prime}(F)=\phi^{\prime \prime}(R) \frac{\mu^{2} F^{2}}{R^{2}}+\phi^{\prime}(R) \frac{\lambda \mu}{R^{3}}-\phi^{\prime \prime}(F) .
$$


Using that $\phi^{\prime \prime}(F)=\mu \phi^{\prime \prime}(R)$ and that $\mu F^{2}-R^{2}=\mu-1=$ $-\lambda,(\mathrm{B} 7)$ can be rearranged to

$$
\begin{aligned}
H^{\prime \prime}(F) & =\frac{\lambda \mu}{R^{3}}\left(-R \phi^{\prime \prime}(R)+\phi^{\prime}(R)\right) \\
& =\frac{1}{\ln (2)} \frac{\lambda \mu}{R^{3}}\left[\frac{R}{1-R^{2}}-\frac{1}{2} \ln \left(\frac{1+R}{1-R}\right)\right] \\
& =\frac{1}{4 \ln (2)} \frac{\lambda \mu}{R^{3}}\left(Z-\frac{1}{Z}-2 \ln (Z)\right),
\end{aligned}
$$

where we set $Z=(1+R) /(1-R)$ and we used that

$$
\frac{4 R}{1-R^{2}}=\frac{(1+R)^{2}-(1-R)^{2}}{(1+R)(1-R)}=Z-\frac{1}{Z}
$$

Finally, we note that $(\lambda \mu) /\left(4 \ln (2) R^{3}\right) \geq 0$ and that, for $Z \geq 1$

$$
\begin{aligned}
Z-\frac{1}{Z}-2 \ln (Z) & =\int_{1}^{Z} \mathrm{~d} z\left(1+\frac{1}{z^{2}}\right)-2 \int_{1}^{Z} \mathrm{~d} z \frac{1}{z} \\
& =\int_{1}^{Z} \mathrm{~d} z\left(1-\frac{1}{z}\right)^{2} \\
& \geq 0
\end{aligned}
$$

which together imply $H^{\prime \prime}(F) \geq 0$.

From (B6), we see that $H^{\prime}(0)=0$, confirming that $F=$ 0 is at least a local extremum. Since $H$ is convex, the only possibility is that $F=0$ is, in fact, the global minimum, in turn implying that $H$ is an increasing function of $F$ over the range $0 \leq F \leq 1$.
[1] C. H. Bennett and G. Brassard, in Proceedings of IEEE International Conference on Computers, Systems and Signal Processing (IEEE, New York, 1984) pp. 175-179.

[2] W. K. Wootters and W. H. Zurek, Nature (London) 299, 802 (1982).

[3] D. Dieks, Phys. Lett. A 92, 271 (1982).

[4] P. W. Shor and J. Preskill, Phys. Rev. Lett. 85, 441 (2000).

[5] C. A. Fuchs, N. Gisin, R. B. Griffiths, C.-S. Niu, and A. Peres, Phys. Rev. A 56, 1163 (1997).

[6] B. Kraus, N. Gisin, and R. Renner, Phys. Rev. Lett. 95, 080501 (2005).

[7] R. Renner, N. Gisin, and B. Kraus, Phys. Rev. A $\mathbf{7 2}$, $012332(2005)$.

[8] G. Smith, J. M. Renes, and J. A. Smolin, Phys. Rev. Lett. 100, 170502 (2008).

[9] H. F. Chau, Phys. Rev. A 66, 060302(R) (2002).

[10] J. Bae and A. Acín, Phys. Rev. A 75, 012334 (2007).

[11] M. Mertz, H. Kampermann, Z. Shadman, and D. Bruß, Phys. Rev. A 87, 042312 (2013).

[12] M. Koashi and J. Preskill, Phys. Rev. Lett. 90, 057902
(2003).

[13] D. Gottesman, H.-K. Lo, N. Lütkenhaus, and J. Preskill, Quantum Inf. Comput. 4, 325 (2004).

[14] M. Koashi, New J. Phys. 11, 045018 (2009).

[15] Ø. Marøy, L. Lydersen, and J. Skaar, Phys. Rev. A 82, 032337 (2010).

[16] H.-W. Li, Z.-Q. Yin, S. Wang, W.-S. Bao, G.-C. Guo, and Z.-F. Han, Quantum Inf. Comput. 11, 937 (2011).

[17] M. Tomamichel, C. C. W. Lim, N. Gisin, and R. Renner, Nat. Commun. 3, 634 (2012).

[18] E. Woodhead, Phys. Rev. A 88, 012331 (2013).

[19] I. Devetak and A. Winter, Proc. R. Soc. A 461, 207 (2005).

[20] E. Biham, M. Boyer, G. Brassard, J. van de Graaf, and T. Mor, Algorithmica 34, 372 (2002).

[21] E. Woodhead and S. Pironio, Article in preparation.

[22] A. Acín, N. Brunner, N. Gisin, S. Massar, S. Pironio, and V. Scarani, Phys. Rev. Lett. 98, 230501 (2007).

[23] M. Christandl, R. König, and R. Renner, Phys. Rev. Lett. 102, 020504 (2009). 